\title{
Guided self-help in a brave new world ${ }^{\dagger}$
}

Jaime Delgadillo

\section{Summary}

Cognitive-behavioural therapy self-help offers an accessible and efficient way to treat common mental disorders. The evidencebased self-help movement has an important foothold in the healthcare arena. This article surveys the emergence of self-help at a particular social and historical junction, and summarises key lessons from experimental and practice-based studies.

\section{Declaration of interest}

None.

\section{Copyright and usage}

(c) The Royal College of Psychiatrists 2018.
Jaime Delgadillo is a lecturer in clinical psychology at the University of Sheffield and a practicing psychotherapist with the National Health Service in England.

At the turn of the 20th century, when psychology was a nascent discipline, Freud famously stated that the objective of psychotherapy was to transform pathological misery into ordinary unhappiness. ${ }^{1}$ Such a glum picture seems fitting against a canvas of Victorian-era moral repression and wet war-paint from continental conflicts that would spill again in the coming decades. At the time, the yoke of human misery that had long been delegated to the clergy was shifting to different patriarchal figures: experts in medicine and psychology. Fast forward a century, and ideas about emotional suffering have changed dramatically. Human misery is seen as a quantifiable aspect of health, mental health professionals are no longer the sole arbiters of expertise and the tools of their trade are now available to the masses. Psychological self-help has come of age in a historical junction where societal norms about individual freedom, social justice and neoliberalism converge. Its narrative is attuned with today's ideals in Western society: misery can be fixed by science, the individual can aspire to live a fulfilling life and the key to unlock happiness is a commodity available in the marketplace.

From a sociological perspective, the broadly defined self-help movement is facilitated by prevailing cultural narratives. But unlike the peer support, coaching and inspirational self-improvement literatures, self-help based on principles of cognitivebehavioural therapy (CBT) gains traction from its impressive scientific credentials. Nearly 40 years ago, Glasgow and Rosen ${ }^{2}$ reviewed over 60 studies and case reports in which self-help manuals based on principles of behaviour therapy were applied to phobias, smoking, childhood behavioural problems and other issues. They concluded that self-help seemed potentially efficacious but further validation was required in clinical populations, with appropriate control conditions, longer follow-up, and attending to predictors of treatment outcome. Since then, numerous studies heeding these recommendations have been documented in over 30 reviews and meta-analyses, covering themes such as (self-administered) bibliotherapy, ${ }^{3}$ guided self-help, ${ }^{4,5}$ technology-assisted self-help ${ }^{6,7}$ and guided self-help to prevent the onset of mental health problems. ${ }^{8}$ There is now compelling evidence from over 50 controlled trials that CBT-based selfhelp interventions are efficacious, particularly in relieving the acute-phase symptoms of depression and anxiety disorders and in preventing - or at least delaying - their onset. However, several important caveats are worth considering.

\footnotetext{
$\dagger$ See pp. 88-95, this issue
}

The efficacy of guided self-help interventions has been shown to be comparable to that of individual CBT for several psychological and somatic problems. ${ }^{6}$ By implication, self-help is highly efficient, considering its low-intensity (brief), low-cost (delivered by trained coaches or lay volunteers), flexible (delivered in person, by internet, telephone or groups) and accessible (didactic) nature. However, pooled effect sizes favouring self-help over other control groups vary widely from small (e.g. $\sim$ Hedges' $g=0.20$ ) to moderate $(\sim g=0.70)$ in different meta-analyses, with typically moderate indices of heterogeneity. From this, we can deduce that the effects of self-help interventions vary considerably, raising the need to understand the factors that might account for such variability.

Some methodological explanations are that employing certain types of control groups (e.g. waitlist), inadequate concealment of random allocation and recruiting participants from community (versus clinical) settings tends to yield more favourable results. This observation has been raised to temper the enthusiasm with which technology-assisted self-help has been promoted, since several trials of internet CBT have tended to recruit participants via social media and estimates from clinical samples show more modest effect sizes. ${ }^{4,7}$ Furthermore, low adherence to internet CBT is increasingly recognised as an obstacle, ${ }^{7}$ which suggests that clinical management is necessary in some cases. The consensus in clinical guidelines is that offering guided self-help is preferable to unsupported self-help, but this view is contested by others, since the type and intensity of support necessary to attain clinical improvement appears to vary across diagnoses. ${ }^{5}$ For example, panic disorder is highly responsive to bibliotherapy, but adjunctive support is indicated for insomnia, binge eating disorder and unipolar mood disorders. Furthermore, emerging studies suggest that minimal levels of support (e.g. brief telephone contact) are comparable and possibly more effective than face-to-face guided self-help. Minimal support plausibly enhances adherence to the treatment model by minimising patient drop-out and therapist-drift into unspecific or supportive counselling. Indeed, recent applications of highly structured, large-group, psychoeducational CBT delivered with minimal interaction between facilitators and participants yielded effect sizes that are comparable to those reported in meta-analyses of guided self-help. ${ }^{9}$

Following the large-scale dissemination of guided self-help in countries like England, Australia and Canada, practice-based studies have generated new insights about its effectiveness and limitations in routine care. Despite the standardised nature of guided self-help, the effectiveness of treatment is partly influenced by the facilitator, ${ }^{10}$ with some attaining exceptional results even if they apply the same self-help model as their peers. ${ }^{9}$ Possible explanations may be differences in competence and treatment adherence among facilitators. Replicated findings in different services have also 
revealed a predictable pattern of treatment response. ${ }^{9,10}$ Patients with early gains (e.g. reliable improvement) during the first four sessions of guided self-help have the best chance of attaining full remission of symptoms, and those who do not improve by session six are most likely to be nonresponders. This observation of an optimal dose of treatment (four to six sessions) can inform clinicians' decisions to rapidly escalate nonresponders to more intensive treatments, or to extend the length of treatment for early responders to maximize their chance of recovery. Although this trial-anderror approach is an efficient way to assess the benefits of selfhelp in individual cases, the advent of personalised and precision medicine research has led to important advances. Replicated studies have found that patients with specific combinations of features (e.g. severe depression plus personality disorder traits and socioeconomic disadvantages) have a high probability of drop-out and poor outcomes in guided self-help interventions, whereas they tend to respond favourably to more intensive psychological therapy. ${ }^{11}$ This evidence suggests that matching patients to treatments (guided self-help versus high-intensity therapy) could be an optimal way to use scarce resources in public healthcare systems. The importance of judicious treatment matching and outcome monitoring is accentuated by the fact that very few controlled trials of selfhelp have followed participants for 12 months or longer. Meta-analytic reviews that favour acute-phase self-help interventions have reported significantly smaller effect sizes at 12 months of followup, ${ }^{4}$ consistent with practice-based evidence that approximately $53 \%$ of guided self-help completers relapse within the first year (mostly within 6 months) of treatment completion. ${ }^{12}$ Taken together, these findings suggest that treatment outcomes could be improved by (a) matching patients to treatments and/or therapists; (b) routinely using outcome measures to monitor progress during the acute phase; and (c) applying interventions to prevent relapse during the continuation phase after initial remission of symptoms.

Based on the current scientific literature, self-help deserves a place at the table of healthcare interventions in a brave new world of evidence-based treatment, public ownership of knowledge and rapidly evolving technologies. It is also clear that self-help has not yet deposed the role of traditional psychotherapy, at least in the treatment of those with more complex conditions, those with recurrent disorders and conditions like anorexia nervosa, post-traumatic stress disorder or obsessive-compulsive disorder. But these two treatment models should be seen as complementary from a population-based and stratified care perspective. In particular, self-help has a unique role to play in the area of prevention, and its potential is yet to be fully realised in supporting the well-being of populations such as students, young people in social care, elderly people, people with chronic illnesses, armed forces, etc. It also has the potential to reach people in need of treatment but who might not otherwise seek formal medical or psychological input. This issue of the British Journal of Psychiatry, for example, features a remarkable study by Williams et $a l,{ }^{13}$ in which lay volunteers offered group self-help interventions for depression in community venues, in a highly effective way. Such interventions have a tremendous effect on access to care and have an important social function in the translation of scientific ideas and destigmatisation of mental health problems.

By the same token, it is prudent to recognise that prevailing cultural ideals also permeate the subtext of self-help interventions which promise to enable people to 'live their life to the full', or to 'beat the blues'. This modern optimism and cultural primacy of the individual are palpable in the new marketplace of (largely unregulated) well-being apps and internet programmes that boldly promise solutions to most human misfortunes. This overoptimistic stance does not seem so pernicious until we realise that it blurs the boundaries between psychopathology and ordinary unhappiness, which is often influenced by wider cultural, relational and socioeconomic determinants. A cautionary tale can be found in the proliferation of commercial mindfulness products that offer solutions to miscellaneous health, interpersonal and occupational problems, commodifying a watered-down version of Eastern philosophy with tenuous links to science. The evidence-based selfhelp movement has undoubtedly stamped an enduring footprint in the field of mental healthcare; its future advancement and integrity hinge on its commitment to scientific rigour, cautiousness and ideological impartiality.

Jaime Delgadillo, PhD, Clinical Psychology Unit, Department of Psychology, University of Sheffield, Sheffield, UK.

Correspondence: Jaime Delgadillo, Clinical Psychology Unit, University of Sheffield, Cathedral Court, Floor F, 1 Vicar Lane, Sheffield S1 1HD, UK.

Email: jaime.delgadillo@nhs.net

First received 17 Sep 2017, accepted 21 Sep 2017

\section{References}

1 Breuer J, Freud S. Studies on hysteria, 1895. In The Standard Edition of the Complete Psychological Works of Sigmund Freud (ed J Strachey): 1-305, Vol. II. Hogarth Press, 1955.

2 Glasgow RE, Rosen GM. Behavioral bibliotherapy: a review of self-help behavior therapy manuals. Psychol Bull 1978; 85: 1-23.

3 Cuijpers P. Bibliotherapy in unipolar depression: a meta-analysis. J Behav Ther Exp Psychiatry 1997; 28: 139-47.

4 Coull G, Morris PG. The clinical effectiveness of CBT-based guided self-help interventions for anxiety and depressive disorders: a systematic review. Psychol Med 2011; 41: 2239-52.

5 Farrand P, Woodford J. Impact of support on the effectiveness of written cognitive behavioural self-help: a systematic review and meta-analysis of randomised controlled trials. Clin Psychol Rev 2013; 33: 182-95.

6 Andersson G, Cuijpers P, Carlbring P, Riper H, Hedman E. Guided internet-based vs. face-to-face cognitive behavior therapy for psychiatric and somatic disorders: a systematic review and meta-analysis. World Psychiatry 2014; 13: 288-95.

7 Karyotaki E, Riper H, Twisk J, Hoogendoorn A, Kleiboer A, Mira A, et al. Efficacy of self-guided internet-based cognitive behavioral therapy in the treatment of depressive symptoms: a meta-analysis of individual participant data. JAMA Psychiatry 2017; 74: 351-9.

8 Cuijpers P, Muñoz RF, Clarke GN, Lewinsohn PM. Psychoeducational treatment and prevention of depression: the 'Coping with Depression' course thirty years later. Clin Psychol Rev 2009; 29: 449-58.

9 Delgadillo J, Kellett S, Ali S, McMillan D, Barkham M, Saxon D, et al. A multiservice practice research network study of large group psychoeducational cognitive behavioural therapy. Behav Res Ther 2016; 87: 155-61.

10 Firth N, Barkham M, Kellett S, Saxon D. Therapist effects and moderators of effectiveness and efficiency in psychological wellbeing practitioners: a multilevel modelling analysis. Behav Res Ther 2015; 69: 54-62.

11 Delgadillo J, Huey D, Bennett H, McMillan D. Case complexity as a guide for psychological treatment selection. J Consult Clin Psychol 2017; 85: 835-53.

12 Ali S, Rhodes L, Moreea O, McMillan D, Gilbody S, Leach C, et al. How durable is the effect of low intensity CBT for depression and anxiety? Remission and relapse in a longitudinal cohort study. Behav Res Ther 2017; 94: 1-8.

13 Williams C, McClay C-A, Matthews L, McConnachie A, Haig C, Walker A, et al. Community-based group guided self-help intervention for low mood and stress: randomised controlled trial. Br J Psychiatry 2018; 212: 88-95. 